\title{
Risk Assessment and Recommendation of Post Exposure Prophylaxis after a Sentinel Case of Rabies in a Tertiary Care Hospital, South India
}

\author{
Haritha Madigubba', Monika², Rahul Dhodapkar ${ }^{2}$, \\ Narayanan Parameswaran ${ }^{3}$ and Apurba Sankar Sastry ${ }^{4 *}$ \\ ${ }^{1}$ Post Doctoral Fellowship in Hospital Infection Control, Jipmer, Puducherry-605006, India \\ ${ }^{2}$ Department of Microbiology, Jipmer, Puducherry-605006, India \\ ${ }^{3}$ Department of Paediatrics, Jipmer, Puducherry-605006, India \\ ${ }^{4}$ Infection control officer, Associate Professor Microbiology Jipmer, \\ Puducherry-605006, India \\ *Corresponding author
}

\section{A B S T R A C T}

Rabies is the oldest zoonotic disease known to mankind. Most of the human cases reported worldwide are due to dog bite, although few cases may be transmitted by

\section{Keywords}

Rhabdoviridae, zoonotic disease, Lyssavirus

\section{Article Info}

Accepted:

20 August 2019 Available Online: 10 September 2019 non-bite exposures. Theoretically high-risk exposures include direct contact of broken skin or mucosa with saliva, tears, oropharyngeal secretions and CSF. Hereby we narrate our risk assessment and post-exposure prophylaxis (PEP) for HCWs at our hospital after a case of rabies was detected. We incorporated our assessment program in the following form: (1) identification of potentially exposed HCWs and laboratory staff, (2) risk analysis by the Hospital infection control committee by using a questionnaire (3) Based on the type of exposure, PEP was recommended to few HCW's and lab staff. 63 persons in total came in contact with the rabies patient, of which 36 were HCW's and 27 were Laboratory staff. All 63 underwent risk assessment, but PEP was recommended for 28/63, who were considered as high risk exposures. After confirming that it is a case of rabies, an integrated attempt was put up by our infection control staff, which culminated in a prompt risk analysis of all those who were potentially exposed. The HCW's were counselled regarding the use of standard precautions while handling the patients and their samples and also to use adequate PPE during performing all the procedures.

\section{Introduction}

Rabies is the oldest zoonotic disease known to mankind. It is an acute, progressive, and fatal encephalomyelitis caused by the Rabies virus (RABV) and other Lyssavirus species of the family Rhabdoviridae. (1). Rabies sustains to pose a major public health challenge in India. Although the exact magnitude of the disease is not known reliably, some studies estimate that 174 lakh persons are bitten and approximately 20000 persons succumb to the disease 
annually. (2) Most of the human cases reported worldwide are due to dog-transmitted rabies. Annually, 61,000 human deaths have been estimated globally, mostly in Asia and Africa. India accounts for one third of the global disease burden.

In India dogs are responsible for about $97 \%$ of human rabies. Human infections from rabies due tonon-bite transmission routes include mucous membrane contamination, aerosols released due to laboratory activities, organ transplantation, and improper inactivation of vaccines. (3)

A category 3 dog bite case was received in our hospital, who had a history of dog bite 1 month prior to admission. Initially on admission he was suspected of acute febrile encephalopathy and was treated for the same. But rabies workup was done for the patient and the serum and CSF antibodies were suspicious for rabies. So there was a fear among the healthcare workers (HCWs) regarding the communication of disease from the infected patient.

Although there has never been a documented evidence of transmission of rabies to HCWs, theoretically high-risk exposures include direct contact of broken skin or mucosa with saliva, tears, oropharyngeal secretions and cerebrospinal fluid. Urine and feces are considered as non-infectious. (4)

Hereby we narrate our risk analysis and postexposure prophylaxis (PEP) for HCWs at our hospital after a case of rabies was detected.

\section{Materials and Methods}

We planned our risk analysis after the affirmation of rabies by RFFIT (Rapid Fluorescent Focus Inhibition Test) in Serum \& CSF (>1024 in Serum \& 256 in CSF).
The present study incorporated the assessment program in the following form: (1) identification of potentially exposed HCWs and laboratory staff; (2) instantaneous alert of these HCWs and staff; (3) risk analysis by the Hospital infection control committee staff by using a questionnaire (4) Based on the type of exposure, PEP was recommended to few HCW's and lab staff who were suspected of having high-risk exposure.

The duration of potential transmission was from the date of admission in our hospital till the time of his death. The patient was on standard precautions, during his hospitalization.

Immediately after the patient was confirmed of having rabies, the Pediatric and Microbiology department chiefs were advised to notify their employees to report for risk assessment. We prepared a questionnaire for the HCW's who documented care for the patient and the laboratory staff who processed the specimens received from that patient. This risk assessment was carried out by the Infection Control Team of JIPMER, to interview and counsel the exposed HCW'S and laboratory staff. The risk assessment form for HCW's and lab staff is as following (Table $1 \mathrm{a}$ and $1 \mathrm{~b}$ ).

In our study, we concluded those as specific high-risk rabies exposures, who had a direct contact with the patient's respiratory secretions, saliva, tears, cerebrospinal fluid, or any laboratory specimens without wearing adequate personal protective equipment. (4). The HCW's who were present around the patient during procedures like ET tube intubation or during suctioning of ET secretions, those were also considered as highrisk rabies exposed, if adequate PPE was not worn. 
Persons identified as having high-risk exposure, Post-Exposure Prophylaxis was recommended to them by the HICC team. The PEP recommended was to receive Rabies cell culture vaccine, IM, 5 doses at $0,3,7,14$ and 28 days. (5)Those who were not at high risk, PEP was not recommended. Supplementary comprehensive counseling was provided to HCWs who had a suspicion of rabies transmission.

\section{Results and Discussion}

Our medical center is a tertiary care teaching hospital. It is an institution of National Importance.

Our patient had interaction with many HCWs, during his 16-day stay in the pediatric intensive care unit.

As shown in Table 2, 63 persons in total came in contact with the rabies patient, of which 36 were HCW's, who provided care to the patient and 27 were Laboratory staff, who were exposed to the patient's specimens. All 63 underwent risk assessment in the form of a questionnaire provided by the HICC team, who addressed all the questions about intimate procurement of rabies from fearful HCWs.

Recommendations for PEP: Those HCW's who had a direct contact with the patient's saliva, respiratory secretions, tears, CSF, laboratory specimens, without adequate PPE i.e not worn at least one of the following like gown, mask, gloves, were considered as potentially high-risk exposures.

In addition, those HCW's who were around the patient during procedures like NG tube insertion, Tracheal tube maintenance, LP, ET tube intubation and suctioning, and were not wearing adequate PPEi.e not worn at least one of the following like gown, mask, gloves, were considered as potentially high-risk exposures.

All the HCW's and the laboratory staff, who were considered as high-risk exposures and who had a potential need for PEP was discussed by the Hospital Infection Control Officer, Senior Virologist and the Pediatrician, before the final decision regarding the PEP recommendation was given.

As shown in Table 2, of the total 63 persons who came in contact with the rabies patient either directly or indirectly, PEP was recommended for 28, who were considered high risk. For 35, PEP was not recommended. No HCWs sustained any bites from our patient. None of the HCW's and lab staff were previously vaccinated for rabies.

Immediately after the confirmation of rabies in the patient, our hospital infection control team screened $63 \mathrm{HCWs}$ at potential risk, of whom 28 persons were recommended to take PEP.

Because non-intact skin or mucosal contact with the patient's secretions, body fluids etc are considered as specific high risk exposures, some of these would have been avertible if the HCWs would have practiced standard precautions during patient care or contaminated medical equipment. (6). Also would have been avoidable, if they had used adequate personal protective equipment when assisting few procedures which can cause aerosolization.

The CDC reported that during 1980-1996, that PEP was given to a mean of 64.6 persons per case (SD, 40.8 persons per case) after potential exposureto rabies. (7). For HCWs, PEP is not simply given after routine healthcare delivery and is warranted after specific risk exposures only. 
Table.1A Rabies Exposure Risk Assessment Form, JIPMER

Name:

Date:

Profession:

Department:

Q1. Did you have any direct physical contact with this patient?

Yes/No

Q2. Were you bitten by this patient?

$\mathrm{Yes} / \mathrm{No}$

Q3. Did you have direct contact with this patient's saliva, respiratory secretions, tears, CSF or laboratory specimens, which are considered infectious for rabies?

Yes/No

(*Note that blood, feces and urine are not considered infectious for rabies)

Q4. Which of the following PPE were you wearing when you had contact with this patient or the patient's body fluids?

maskGown

Q5. Did any of the above mentioned infectious materials enter into your eyes, nose, mouth or fresh open wound?

Yes/No

Q6. Did you perform or were you in the patient's room when following procedures were performed?

\begin{tabular}{|c|c|c|c|c|c|c|}
\hline \multirow[t]{2}{*}{ Procedures } & \multirow[t]{2}{*}{ Performed } & \multirow{2}{*}{$\begin{array}{l}\text { Not } \\
\text { performed, } \\
\text { Present } \\
\text { nearby }\end{array}$} & \multicolumn{4}{|c|}{ PPE used during procedure } \\
\hline & & & Gloves & Gown & Mask & Goggles \\
\hline $\begin{array}{l}\text { NG tube } \\
\text { insertion }\end{array}$ & & & & & & \\
\hline $\begin{array}{l}\text { Tracheal } \\
\text { tube } \\
\text { maintenance }\end{array}$ & & & & & & \\
\hline $\begin{array}{l}\text { Lumbar } \\
\text { puncture }\end{array}$ & & & & & & \\
\hline Intubation & & & & & & \\
\hline $\begin{array}{l}\text { ET } \\
\text { suctioning }\end{array}$ & & & & & & \\
\hline
\end{tabular}

Q8. Did you sustain a needle stick injury after it was or may have been in contact with the patients saliva, respiratory secretions, or CSF?

Yes/No

Q9. Have you been previously immunized against rabies?

Yes/No

ADVICE by HICC team about PEP: PEP Recommended $\square$ PEP Not Recommended

Signature of HICC team

healthcare worker

Signature of the 
Table.1B Rabies risk assessment form for laboratory staff, JIPMER

Name:

Date:

profession:

Department:

Q1. What sample did you handle? (tick the answer)

A1. Blood/ Serum/ CSF/ Urine/ Tracheal aspirate/ Sputum/ Any other (mention here-)

Q2. What process of sample did you perform in relation to the above sample?

A2. 1. Received in reception

2. aliquoted

3. performed test (if yes, give details of the test-)

4. handled discarded sample

Q3. Specify which procedures were performed in the test performed?

A3. 1. Centrifugation

2. vortex

3. pipetting

4. culture inoculation

5. smear preparation

6. loading in automated instrument

Q4. What PPE did you use while performing the above procedures?
A4. 1. Gloves
2. Mask
3. N95 mask

4. Gown

5. goggles

Q5. How did you discard the specimen after the test?

PEP Advice by HICC: PEP Recommended

PEP not recommended

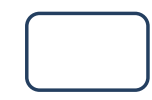

Signature of HICC staff

Signature of Healthcare worker 
Table.2

\begin{tabular}{|c|c|c|c|c|}
\hline Number & Profession & Department & Type of exposure & PEP \\
\hline 1. & Doctor & Paediatrics & $\begin{array}{l}\text { ET suctioning, adequate PPE } \\
\text { not worn }\end{array}$ & Recommended \\
\hline 2. & Junior Resident & Paediatrics & $\begin{array}{l}\text { ET suctioning, NG tube } \\
\text { insertion, LP, Intubation, PPE- } \\
\text { only gloves worn, adequate } \\
\text { PPE not worn }\end{array}$ & Recommended \\
\hline 3. & Junior Resident & PICU-Paediatrics & $\begin{array}{l}\text { ET suctioning, NG tube } \\
\text { insertion, LP, Intubation, PPE- } \\
\text { only gown worn, adequate PPE } \\
\text { not worn }\end{array}$ & Recommended \\
\hline 4. & Junior Resident & Paediatrics & $\begin{array}{l}\text { ET suctioning, NG tube } \\
\text { insertion, LP, Intubation, PPE- } \\
\text { only gown worn, adequate PPE } \\
\text { not worn }\end{array}$ & Recommended \\
\hline 5. & Junior Resident & Paediatrics & $\begin{array}{l}\text { Tracheal tube maintenance \& } \\
\text { ET suctioning, adequate PPE } \\
\text { not worn }\end{array}$ & Recommended \\
\hline 6 & Nursing Officer & $\begin{array}{l}\text { PICU-Nursing } \\
\text { Section }\end{array}$ & $\begin{array}{l}\text { Present nearby during ET } \\
\text { suctioning, adequate PPE not } \\
\text { worn }\end{array}$ & Recommended \\
\hline 7 & Junior Resident & Paediatrics & $\begin{array}{l}\text { Tracheal tube maintenance \& } \\
\text { ET suctioning, adequate PPE } \\
\text { not worn }\end{array}$ & Recommended \\
\hline 8 & Nursing Officer & $\begin{array}{l}\text { PICU-Nursing } \\
\text { Section }\end{array}$ & $\begin{array}{l}\text { Tracheal tube maintenance \& } \\
\text { ET suctioning, adequate PPE } \\
\text { not worn }\end{array}$ & Recommended \\
\hline 9 & Junior Resident & Paediatrics & ET suctioning, PPE not worn & Recommended \\
\hline 10 & Junior Resident & Paediatrics & $\begin{array}{l}\text { Tracheal tube maintenance \& } \\
\text { ET suctioning, adequate PPE } \\
\text { not worn }\end{array}$ & Recommended \\
\hline 11 & Nursing Officer & $\begin{array}{l}\text { PICU-Nursing } \\
\text { Section }\end{array}$ & $\begin{array}{l}\text { Present nearby during ET } \\
\text { suctioning, adequate PPE not } \\
\text { worn }\end{array}$ & Recommended \\
\hline 12 & Nursing Officer & $\begin{array}{l}\text { PICU-Nursing } \\
\text { Section }\end{array}$ & $\begin{array}{l}\text { Present nearby during ET } \\
\text { suctioning \& intubation } \\
\text { adequate PPE not worn }\end{array}$ & Recommended \\
\hline 13 & Nursing Officer & $\begin{array}{l}\text { PICU-Nursing } \\
\text { Section }\end{array}$ & $\begin{array}{l}\text { Tracheal tube maintenance- } \\
\text { adequate PPE worn \& } \\
\text { ET suctioning- adequate PPE } \\
\text { not worn }\end{array}$ & Recommended \\
\hline 14 & Nursing Officer & $\begin{array}{l}\text { Paediatrics- } \\
\text { Nursing Section }\end{array}$ & $\begin{array}{l}\text { Present nearby during ET } \\
\text { suctioning \& intubation, } \\
\text { adequate PPE worn }\end{array}$ & $\begin{array}{l}\text { Not } \\
\text { Recommended }\end{array}$ \\
\hline 15 & Nursing Officer & $\begin{array}{l}\text { PICU-Nursing } \\
\text { Section }\end{array}$ & $\begin{array}{l}\text { ET suctioning, NG tube } \\
\text { insertion, Intubation, adequate } \\
\text { PPE worn }\end{array}$ & $\begin{array}{l}\text { Not } \\
\text { Recommended }\end{array}$ \\
\hline
\end{tabular}




\begin{tabular}{|c|c|c|c|c|}
\hline 16 & Senior Resident & PICU-Paediatrics & $\begin{array}{l}\text { Present nearby during ET } \\
\text { suctioning, adequate PPE not } \\
\text { worn }\end{array}$ & Recommended \\
\hline 17 & Senior Resident & PICU-Paediatrics & $\begin{array}{l}\text { Present nearby during ET } \\
\text { suctioning, adequate PPE not } \\
\text { worn }\end{array}$ & Recommended \\
\hline 18 & Professor & Paediatrics & $\begin{array}{l}\text { Present nearby during ET } \\
\text { suctioning, adequate PPE not } \\
\text { worn }\end{array}$ & Recommended \\
\hline 19 & Intern & PICU-Paediatrics & $\begin{array}{l}\text { ET suctioning performed, } \\
\text { adequate PPE worn }\end{array}$ & $\begin{array}{l}\text { Not } \\
\text { Recommended }\end{array}$ \\
\hline 20 & $\begin{array}{l}\text { Senior Nursing } \\
\text { Officer }\end{array}$ & $\begin{array}{l}\text { PICU-Nursing } \\
\text { Section }\end{array}$ & $\begin{array}{l}\text { Present nearby during ET } \\
\text { suctioning \& tracheal tube } \\
\text { maintenance, adequate PPE not } \\
\text { worn }\end{array}$ & Recommended \\
\hline 21 & $\begin{array}{l}\text { Senior Nursing } \\
\text { Officer }\end{array}$ & $\begin{array}{l}\text { PICU-Nursing } \\
\text { Section }\end{array}$ & $\begin{array}{l}\text { Not performed any procedures, } \\
\text { not handled any specimen }\end{array}$ & $\begin{array}{l}\text { Not } \\
\text { Recommended }\end{array}$ \\
\hline 22 & Junior Resident & Paediatrics & $\begin{array}{l}\text { ET suctioning performed, } \\
\text { adequate PPE worn }\end{array}$ & $\begin{array}{l}\text { Not } \\
\text { Recommended }\end{array}$ \\
\hline 23 & DRL & Paediatrics & $\begin{array}{l}\text { Present nearby during all the } \\
\text { procedures, adequate PPE not } \\
\text { worn }\end{array}$ & Recommended \\
\hline 24 & Senior Resident & PICU-Paediatrics & $\begin{array}{l}\text { ET suctioning performed, } \\
\text { adequate PPE not worn }\end{array}$ & Recommended \\
\hline 25 & Senior Resident & PICU-Paediatrics & $\begin{array}{l}\text { Present nearby during all the } \\
\text { procedures, adequate PPE not } \\
\text { worn }\end{array}$ & Recommended \\
\hline 26 & Nursing Officer & $\begin{array}{l}\text { PICU-Nursing } \\
\text { Section }\end{array}$ & $\begin{array}{l}\text { Tracheal tube maintenance \& } \\
\text { ET suctioning, adequate PPE } \\
\text { worn }\end{array}$ & $\begin{array}{l}\text { Not } \\
\text { Recommended }\end{array}$ \\
\hline 27 & Nursing Officer & $\begin{array}{l}\text { PICU-Nursing } \\
\text { Section }\end{array}$ & $\begin{array}{l}\text { Tracheal tube maintenance \& } \\
\text { ET suctioning, adequate PPE } \\
\text { worn }\end{array}$ & $\begin{array}{l}\text { Not } \\
\text { Recommended }\end{array}$ \\
\hline 28 & Junior Resident & Paediatrics & $\begin{array}{l}\text { ET suctioning performed, } \\
\text { adequate PPE not worn }\end{array}$ & Recommended \\
\hline 29 & DRL & PICU-Paediatrics & $\begin{array}{l}\text { Not performed any procedures, } \\
\text { not handled any specimen }\end{array}$ & $\begin{array}{l}\text { Not } \\
\text { Recommended }\end{array}$ \\
\hline 30 & DRL & PICU-Paediatrics & $\begin{array}{l}\text { Not performed any procedures, } \\
\text { not handled any specimen }\end{array}$ & $\begin{array}{l}\text { Not } \\
\text { Recommended }\end{array}$ \\
\hline 31 & DRL & PICU-Paediatrics & $\begin{array}{l}\text { Not performed any procedures, } \\
\text { not handled any specimen }\end{array}$ & $\begin{array}{l}\text { Not } \\
\text { Recommended }\end{array}$ \\
\hline 32 & DRL & PICU-Paediatrics & $\begin{array}{l}\text { Not performed any procedures, } \\
\text { not handled any specimen }\end{array}$ & $\begin{array}{l}\text { Not } \\
\text { Recommended }\end{array}$ \\
\hline 33 & Nursing Officer & $\begin{array}{l}\text { PICU-Nursing } \\
\text { Section }\end{array}$ & $\begin{array}{l}\text { Present nearby during ET } \\
\text { suctioning, adequate PPE not } \\
\text { worn }\end{array}$ & Recommended \\
\hline 34 & Nursing Officer & $\begin{array}{l}\text { PICU-Nursing } \\
\text { Section }\end{array}$ & $\begin{array}{l}\text { Present nearby during ET } \\
\text { suctioning \& tracheal tube } \\
\text { maintenance, adequate PPE not }\end{array}$ & Recommended \\
\hline
\end{tabular}




\begin{tabular}{|c|c|c|c|c|}
\hline & & & worn & \\
\hline 35 & Nursing Officer & $\begin{array}{l}\text { PICU-Nursing } \\
\text { Section }\end{array}$ & $\begin{array}{l}\text { Present nearby during ET } \\
\text { suctioning, adequate PPE not } \\
\text { worn }\end{array}$ & Recommended \\
\hline 36 & Nursing Officer & $\begin{array}{l}\text { PICU-Nursing } \\
\text { Section }\end{array}$ & $\begin{array}{l}\text { Present nearby during ET } \\
\text { suctioning, adequate PPE not } \\
\text { worn }\end{array}$ & Recommended \\
\hline 37 & SRF & Microbiology & Handled blood (non infectious) & $\begin{array}{l}\text { Not } \\
\text { Recommended }\end{array}$ \\
\hline 38 & Lab Technician & Microbiology & $\begin{array}{l}\text { Handled blood (non } \\
\text { infectious)- } \quad \text { centrifugation } \\
\text {,pipetting \& loading }\end{array}$ & $\begin{array}{l}\text { Not } \\
\text { Recommended }\end{array}$ \\
\hline 39 & Lab Technician & Microbiology & $\begin{array}{l}\text { Handled samples after DNA } \\
\text { extraction (non-infectious) }\end{array}$ & $\begin{array}{l}\text { Not } \\
\text { Recommended }\end{array}$ \\
\hline 40 & Lab Technician & Microbiology & $\begin{array}{l}\text { Handled blood \& urine (non } \\
\text { infectious) \& CSF / } \\
\text { centrifugation,pipetting\& } \\
\text { loading }\end{array}$ & Recommended \\
\hline 41 & $\begin{array}{l}\text { Medical Lab } \\
\text { Technologist }\end{array}$ & Microbiology & $\begin{array}{l}\text { Handled blood (non infectious) } \\
\text { / centrifugation,pipetting \& } \\
\text { loading }\end{array}$ & $\begin{array}{l}\text { Not } \\
\text { Recommended }\end{array}$ \\
\hline 42 & Lab Technician & Microbiology & $\begin{array}{l}\text { Received CSF specimen, worn } \\
\text { gloves }\end{array}$ & $\begin{array}{l}\text { Not } \\
\text { Recommended }\end{array}$ \\
\hline 43 & Lab Technician & Microbiology & $\begin{array}{l}\text { Handled CSF/Tracheal } \\
\text { aspirate \& sputum / pipetting \& } \\
\text { loading, worn adequate PPE, } \\
\text { adquate PPE worn }\end{array}$ & $\begin{array}{l}\text { Not } \\
\text { Recommended }\end{array}$ \\
\hline 44 & $\begin{array}{l}\text { Medical Lab } \\
\text { Technologist }\end{array}$ & Microbiology & $\begin{array}{l}\text { Handled CSF, Tracheal aspirate } \\
\& \text { urine (non infectious) } \\
\text { centrifugation,pipetting\& } \\
\text { loading, adquate PPE worn }\end{array}$ & $\begin{array}{l}\text { Not } \\
\text { Recommended }\end{array}$ \\
\hline 45 & $\begin{array}{l}\text { Technical } \\
\text { Officer }\end{array}$ & Microbiology & $\begin{array}{l}\text { Handled blood (non infectious) } \\
\& \text { CSF / centrifugation } \\
\text {,pipetting \& loading, adquate } \\
\text { PPE worn }\end{array}$ & $\begin{array}{l}\text { Not } \\
\text { Recommended }\end{array}$ \\
\hline 46 & $\begin{array}{l}\text { Medical Lab } \\
\text { Technologist }\end{array}$ & Microbiology & $\begin{array}{l}\text { Handled blood (non infectious) } \\
\& \text { CSF / centrifugation \& } \\
\text { loading, adequate PPE not } \\
\text { worn }\end{array}$ & Recommended \\
\hline 47 & Lab Technician & Microbiology & $\begin{array}{l}\text { Handled blood (non infectious) } \\
\text { / centrifugation \& loading }\end{array}$ & $\begin{array}{l}\text { Not } \\
\text { Recommended }\end{array}$ \\
\hline 48 & Lab Technician & Microbiology & $\begin{array}{l}\text { Handled blood (non infectious) } \\
\text { / centrifugation \& loading }\end{array}$ & $\begin{array}{l}\text { Not } \\
\text { Recommended }\end{array}$ \\
\hline 49 & $\begin{array}{l}\text { Technical } \\
\text { Officer }\end{array}$ & Microbiology & $\begin{array}{l}\text { Handled Tracheal aspirate \& } \\
\text { sputum / Culture \& smear } \\
\text { preparation, worn gloves and } \\
\text { mask }\end{array}$ & $\begin{array}{l}\text { Not } \\
\text { Recommended }\end{array}$ \\
\hline 50 & $\begin{array}{l}\text { Medical Lab } \\
\text { Technologist }\end{array}$ & Microbiology & $\begin{array}{l}\text { Handled } \quad \text { blood (non } \\
\text { infectious), Tracheal aspirate \& }\end{array}$ & $\begin{array}{l}\text { Not } \\
\text { Recommended }\end{array}$ \\
\hline
\end{tabular}




\begin{tabular}{|c|c|c|c|c|}
\hline & & & $\begin{array}{l}\text { sputum, worn only gloves, } \\
\text { adequate PPE not worn }\end{array}$ & \\
\hline 51 & $\begin{array}{l}\text { B.Sc(MLT) } \\
\text { Intern }\end{array}$ & Microbiology & $\begin{array}{l}\text { Handled blood (non infectious) } \\
\& \text { CSF / Culture \& smear } \\
\text { preparation }\end{array}$ & $\begin{array}{l}\text { Not } \\
\text { Recommended }\end{array}$ \\
\hline 52 & M.Sc Student & Microbiology & $\begin{array}{l}\text { Handled CSF (infectious) \& } \\
\text { urine (non infectious) } \\
\text { centrifugation,pipetting\& } \\
\text { loading, worn adequate PPE }\end{array}$ & $\begin{array}{l}\text { Not } \\
\text { Recommended }\end{array}$ \\
\hline 53 & $\begin{array}{l}\text { B.Sc(MLT) } \\
\text { Intern }\end{array}$ & Microbiology & $\begin{array}{l}\text { Handled CSF,Tracheal aspirate } \\
\text { (infectious) \& urine (non } \\
\text { infectious) } \\
\text { centrifugation,pipetting\& } \\
\text { loading,-worn adequate PPE }\end{array}$ & $\begin{array}{l}\text { Not } \\
\text { Recommended }\end{array}$ \\
\hline 54 & DRL & Microbiology & $\begin{array}{l}\text { Received samples in reception, } \\
\text { worn adequate PPE }\end{array}$ & $\begin{array}{l}\text { Not } \\
\text { Recommended }\end{array}$ \\
\hline 55 & $\begin{array}{l}\text { Research } \\
\text { Assistant }\end{array}$ & Microbiology & $\begin{array}{l}\text { Handled blood (non infectious) } \\
\& \text { CSF / centrifugation } \\
\text {,pipetting \& loading, adequate } \\
\text { PPE not worn }\end{array}$ & Recommended \\
\hline 56 & Lab Technician & Microbiology & Handled blood (non infectious) & $\begin{array}{l}\text { Not } \\
\text { Recommended }\end{array}$ \\
\hline 57 & Junior Resident & Microbiology & $\begin{array}{l}\text { Not handled any samples } \\
\text { directly, handled the culture } \\
\text { plates of the sample }\end{array}$ & $\begin{array}{l}\text { Not } \\
\text { Recommended }\end{array}$ \\
\hline 58 & Junior Resident & Microbiology & $\begin{array}{l}\text { Not handled any samples } \\
\text { directly, handled the culture } \\
\text { plates of the sample }\end{array}$ & $\begin{array}{l}\text { Not } \\
\text { Recommended }\end{array}$ \\
\hline 59 & Junior Resident & Microbiology & $\begin{array}{l}\text { Not handled any samples } \\
\text { directly, handled the culture } \\
\text { plates of the sample }\end{array}$ & $\begin{array}{l}\text { Not } \\
\text { Recommended }\end{array}$ \\
\hline 60 & Junior Resident & Microbiology & $\begin{array}{l}\text { Not handled any samples } \\
\text { directly, handled the culture } \\
\text { plates of the sample }\end{array}$ & $\begin{array}{l}\text { Not } \\
\text { Recommended }\end{array}$ \\
\hline 61 & Faculty & Microbiology & $\begin{array}{l}\text { Not handled any samples } \\
\text { directly, handled the culture } \\
\text { plates of the sample }\end{array}$ & $\begin{array}{l}\text { Not } \\
\text { Recommended }\end{array}$ \\
\hline 62 & Faculty & Microbiology & $\begin{array}{l}\text { Not handled any samples } \\
\text { directly, handled the culture } \\
\text { plates of the sample }\end{array}$ & $\begin{array}{l}\text { Not } \\
\text { Recommended }\end{array}$ \\
\hline 63 & Faculty & Microbiology & Not handled any samples & $\begin{array}{l}\text { Not } \\
\text { Recommended }\end{array}$ \\
\hline
\end{tabular}

(*ET-endotracheal Tube, **NG- Nasogastric, ***LP-Lumbar Puncture)

Considering rabies as a differential diagnosis in the early stages of the disease, following adequate personal protective barriers while handing the patient as well as the patient samples, and thorough risk analysis of the exposed persons can help to avoid 
recommending unnecessary PEP.

In our hospital, $44.4 \%$ of the HCW's who were screened, were recommended to take PEP. Our PEP recommendation rate was comparable to the previous studies done in similar settings after a transplant exposure to rabies, whose PEP coverage in HCW's was reported as $44-50 \%$. (8-15).In few cases of transplanted corneas, where there were only a few HCW's who were exposed, PEP was given to all of them after the confirmation of rabies. Contrary to this, in a case of nontransplant patient, HCW's with even low and no risk received PEP, even though it was recommended only for high risk exposures. (16).There were 2 other reports from California which occurred in the same year. One case was diagnosed postmortem, after which $72 \mathrm{HCW}$ 's received PEP. Another was an antemortem case, in which only $1 \mathrm{HCW}$ received the PEP. (17).

Only 1 report of pre exposure prophylaxis was available for a patient whose rabies was diagnosed ante mortem, in which 3 pathologists received the prophylaxis after performing autopsy. (18). As rabies usually causes severe neurological complications and fatal infection, the use of excessive PEP may be considered among HCW's, as they may have a fear of transmission of disease during the care of the patient. This may also lead to disproportionate deviation from the PEP guidelines. (19, 20). The staff from the Hospital Infection Control clarified all the queries raised by HCWs in an objective and timely manner. In addition to this, they also provided comprehensive education regarding the risks of rabies transmission and the use of PEP for all those HCWs and the laboratory staff who were considered as high risk exposures.

In summary, after confirming that it is a case of rabies, an integrated attempt was put up by our infection control staff, which culminated in a prompt risk analysis of all those who were potentially exposed i.e high HCWs and the laboratory staff. Our team figured out and counseled $63 \mathrm{HCW}$ 's who were potentially exposed, to alleviate their fear regarding the nosocomial spread of rabies to them. A total of $28(44.4 \%)$ HCWs were recommended to receive $\mathrm{PEP}$. We also counselled the HCW's regarding the use of standard precautions while handling the patients and their samples and also to use adequate PPE during performing all the procedures. As suggested in the literatures and also from our own experience, human-to-human transmission of rabies is very rare. Therefore a reactionary approach should be applicable for determining which HCWs should receive PEP after caring for a patient with rabies.

\section{References}

1. Anderson LJ, Williams LP, Layde JB, Dixon FR, Winkler WG. Nosocomial rabies: investigation of contacts of human rabies cases associated with a corneal transplant. Am J Public Health 1984; 74:370-2.

2. Anderson LJ, Winkler WG, Vernon AA, Helmick CG, Roberts MR. Prophylaxis for persons in contact with patients who have rabies. N Engl J Med, 1980; 302: 967-8.

3. Centers for Disease Control and Prevention (CDC). Human rabies acquired outside the United States from a dog bite. MMWR Morb Mortal Wkly Rep 1981; 30:537-40.

4. Centers for Disease Control and Prevention (CDC). Human rabies - California, 1995. MMWR Morb Mortal Wkly Rep 1996; 45:353-6.

5. Centers for Disease Control and Prevention (CDC). Human rabies - Florida, 1996. MMWR Morb Mortal Wkly Rep 1996; 45:719-20, 727.

6. Centers for Disease Control and Prevention (CDC). Human-to-human transmission of 
rabies by a corneal transplant-Idaho. 13. Gode GR, Bhide NK. Two rabies deaths MMWR Morb Mortal Wkly Rep 1979; 28:109-11. after corneal grafts from one donor. Lancet $1988 ; 2: 791$.

7. Centers for Disease Control and Prevention 14. Hoffer J. Prophylaxis in contacts of patients (CDC). Human-to-human transmission of rabies by a corneal transplant-France. MMWR Morb Mortal Wkly Rep 1980; 29:25-6.

8. Centers for Disease Control and Prevention (CDC). Human-to-human transmission of 16 rabies via corneal transplant-Thailand. MMWR Morb Mortal Wkly Rep 1981; 30:473-4.

9. Centers for Disease Control and Prevention (CDC). Imported human rabies. MMWR Morb Mortal Wkly Rep 1983; 32:78-80, 85-6.

10. Centers for Disease Control and Prevention (CDC). Investigation of rabies infections in organ donor and transplant recipientsAlabama, Arkansas, Oklahoma, and Texas, 2004. MMWR Morb Mortal Wkly Rep 2004; 53:586-9.

with rabies. N Engl J Med, 1979; 301: 1451.

15. Javadi MA, Fayaz A, Mirdehghan SA, Ainollahi B. Transmission of rabies by corneal graft. Cornea 1996; 15:431-3.

16. Kan VL, Joyce P, Benator D, Agnes K, Gill J, Irmler M, Clark A, Giannakos G, Gabourel A, Gordin FM. Risk assessment for healthcare workers after a sentinel case of rabies and review of the literature. Clinical Infectious Diseases. 2014 Oct 28;60(3):341-8.

17. Mani RS, Madhusudana SN. Laboratory diagnosis of human rabies: recent advances. The Scientific World Journal. 2013; 2013.

18. National Guidelines on Rabies Prophylaxis, NATIONAL CENTRE FOR DISEASE CONTROL(Directorate General of Health Services)hp://www.ncdc.gov.in 2015.

11. Centers for Disease Control and Prevention 19. Singh R, Singh KP, Saminathan M, (CDC). Update: investigation of rabies infections in organ donor and transplant recipients_-Alabama, Arkansas, Oklahoma, and Texas, 2004. MMWR Morb Mortal Wkly Rep 2004; 53:615-6. Vineetha S, GB MR, Maity M, Cherian S, Dhama K. Rabies, a vaccine preventable disease: Current status, epidemiology, pathogenesis, prevention and control with special reference to India.

12. Dutta JK. Rabies transmission by oral and 20. Weber DJ, Rutala WA. Risks and other non-bite routes. J Indian Med Assoc 1998; 96:359. prevention of nosocomial transmission of rare zoonotic diseases. Clin Infect Dis 2001; 32:446-56.

\section{How to cite this article:}

Haritha Madigubba, Monika, Rahul Dhodapkar, Narayanan Parameswaran and Apurba Sankar Sastry . 2019. Risk Assessment and Recommendation of Post Exposure Prophylaxis after a Sentinel Case of Rabies in a Tertiary Care Hospital, South India. Int.J.Curr.Microbiol.App.Sci. 8(09): 1807-1817. doi: https://doi.org/10.20546/ijcmas.2019.809.209 